\title{
EL AZAR OBJETIVO COMO MEDIDA MATEMÁTICA DE DESORDEN
}

\author{
SERGIO MARTÍNEZ
}

INSTTTUTO DE INUESTTGACIONES FILOSÓFICAS

UnIVersidad Nacional Autónoma de MÉXico

1. Introducción. La cuestión de si el azar existe como una propiedad objetiva de sistemas físicos, esto es, como una propiedad de sistemas o procesos que no es un mero producto de nuestra ignorancia sobre aspectos del mundo físico, es un problema íntimamente ligado con una serie de cuestiones controversiales en los fundamentos de la ciencia. El problema de qué es una ley fundamental de la naturaleza se ve de manera diferente si estamos dispuestos a extender la idea de ley fundamental a procesos azarosos. El problema de la interpretación de las probabilidades es en gran medida la dificultad de caracterizar una noción de azar objetivo que pueda servir de base para la interpretación de las diferentes aplicaciones del cálculo de las probabilidades. Finalmente, la cuestión de la existencia del azar objetivo está íntimamente relacionada con varias discusiones acerca del problema del "realismo" en la moderna filosofía de la ciencia, por ejemplo, a través de las implicaciones que la existencia de un azar objetivo tiene para argumentos realistas basados en análisis de la presencia de una "causa común".

La dificultad primera que encuentra todo intento por clarificar la noción de azar objetivo es que no parece haber un lenguaje filosófico establecido que nos permita enfocar la pregunta bajo un lente lo suficientemente analítico. Esto, en gran medida, creo yo, refleja los presupuestos de un empirismo tradicional que de hecho excluyen la posibilidad de, incluso, poder formular claramente la pregunta. En este trabajo quiero exponer uno de estos presupuestos y hacer ver cómo este presupuesto funciona evitando una posible formulación de la idea de azar objetivo.

El presupuesto en cuestión consiste en la suposición de que el concepto de azar objetivo puede capturarse a través de una medida matemática del "desorden" de sucesiones numéricas que pueden identificarse con sucesiones de resultados observacionales de procesos físicos. Hay diferentes teorías matemáticas que pretenden caracterizar el azar como un concepto matemático de desorden. Sin embargo, para nuestros propósitos estas teorías pueden cla- 
sificarse en dos grandes grupos. Por un lado tenemos teorías matemáticas del azar que definen el azar como una propiedad de sucesiones infinitas. Por otro lado tenemos teorías matemáticas que conciben el azar como una propiedad de sucesiones finitas. Formularemos aquí las dificultades de fondo con ambos tipos de propuesta.

El presupuesto que nos interesa tiene históricamente su origen en las interpretaciones de la probabilidad por frecuencias relativas que tratan de definir la probabilidad como una propiedad empírica objetiva de sistemas físicos. Es por esto que, después de clarificar nuestro proyecto en la próxima sección, haremos ver en la tercera sección cómo el presupuesto en cuestión surge y puede delinearse claramente a partir de cierto tipo de intepretaciones de la probabilidad por frecuencias relativas. En la cuarta sección hago ver cómo el desarrollo de teorías matemáticas de la complejidad de una sucesión no resuelve el problema, aunque sí nos permite percibir más claramente las limitaciones del presupuesto que nos ocupa.

2. La definición de azar objetivo. Un ejemplo paradigmático de un proceso azaroso es el lanzamiento de una moneda sobre una superficie lisa. El resultado del lanzamiento puede ser cara o cruz y la descripción del proceso se da por medio de una función (distribución) probabilista que asigna probabilidades a cada uno de los dos posibles resultados, válidas para cada lanzamiento posible. En este caso es usual pensar que el azar no es objetivo ya que, por lo menos en principio, podríamos fijar las condiciones iniciales con gran precisión. $\mathrm{Si}$ conociéramos las fuerzas que actúan en el proceso podríamos determinar una función determinista, que asignaría un lugar en el espacio para cada tiempo, lo cual nos permitiría conocer el resultado final con certeza.

Esta tesis reduccionista del azar físico ha sido defendida por muchos físicos y filósofos que se han preocupado del azar. ${ }^{1}$ Hay sin embargo presuposiciones implícitas cuestionables detrás de esta tesis reduccionista del azar físico. ¿A

1 Ver por ejemplo Popper (1962), Stegmüller (1973). Podría pensarse que Popper en (1982) es una excepción importante. Popper, sin embargo, sigue compartiendo demasiado con los proponentes de la tesis reduccionista como para tomarlo como un defensor de una tesis diferente. Aunque Popper sostiene que hay azar objetivo, la manera en la que él concibe este azar objetivo parte de una cierta concepción del azar que es esencialmente la misma que la de otros defensores de la tesis reduccionista clásica. Popper concibe la objerividad del azar como una consecuencia de la imposibilidad para un ser humano de determinar condicioncs iniciales con precisión matemática (Popper 1982 p. 34). Esta manera de concebir el azar objetivo trivializa el problema y confunde una serie de cuestiones que deben mantenerse separadas. Popper no sólo confunde el problema epistemológico de la predecibilidad con el problema metafísico del determinismo, sino también tiene el defecto de hacer a un ladu :no de los problemas más importantes en los fundamentos de la teoría clásica de la mecánica estadística: cómo reconciliar la macro-descripción azarosa (sujeta a leyes probabilistas) de una muestra de gas, por ejemplo, con el movimiento supuestamente determinista de las moléculas a nivel microscópico (véase Earman 1986, cap. II). 
qué nos referimos cuando decimos que una descripción determinista es posible? Es plausible que para Dios todo sea presente e inmediato, y que, independientemente de la naturaleza del proceso dinámico en cuestión, para Dios la precisión requerida por la tesis reduccionista arriba ejemplificada siempre sea alcanzable. Pero esto reduciría la tesis reduccionista a una tesis metafísica dificilmente sostenible. Incluso si asumiéramos que Dios está limitado a pensar utilizando funciones matemáticas, la tesis reduccionista sería trivialmente verdadera (como lo enfatizaba Russell) si no tomamos en consideración las restricciones impuestas en el universo físico por las leyes de la naturaleza que se formulan a través de teorías específicas.

Por ejemplo, supongamos que el universo consiste de partículas que se mueven en el espacio de la manera más complicada que nos podamos imaginar, pero de tal manera que las partículas ocupan siempre un lugar en un tiempo dado. Supongamos además (como es usual) que el estado de las partículas está dado por sus coordenadas de posición $x, y, z$. Es un hecho matemático que existen funciones $x_{t}=f_{1}(t), y_{t}=f_{2}(t), z_{t}=f_{3}(t)$. Si todo lo que el determinismo requiriera es la existencia matemática de funciones que describen el movimiento de las partículas, entonces el universo sería trivialmente determinista. Es posible, sin embargo, que la función $E=\left(f_{1}, f_{2}, f_{3}\right)$ no sea físicamente posible en el sentido de que no satisface ciertos requisitos mínimos impuestos por leyes de la naturaleza. En este caso, si el mundo físico es o no determinista es un problema no trivial.

Una manera de definir más precisamente lo que es un proceso físico objetivamente azaroso podría partir de la siguiente idea: un proceso físico es objetivamente azaroso si no hay una teoría posible de la física, esto es, un modelo matemático que satisfaga ciertos requisitos mínimos de adecuación, en la cual el proceso se describa por medio de una función determinista. Más precisamente, puesto que toda teoría posible de la física genera el conjunto de funciones $F(T)$ que son soluciones de las ecuaciones diferenciales básicas de la teoría, podemos reformular la definición anterior como sigue: diremos que un proceso físico descrito por una función probabilista $f \in F(T)$ es objetivamente azaroso si no existe otra teoría $T^{\circ}$ (modelo matemático) físicamente adecuada, y una función $f^{\prime} \in F\left(T^{\circ}\right)$ que describa el proceso de manera determinista. Nótese que no estamos diciendo que el proceso no es objetivamente azaroso si matemáticamente existe una función que describe deterministamente el proceso. Como hemos visto anteriormente esto casi siempre es posible.

Esta manera de reformular la tesis reduccionista parece que nos lleva del brasero a las brasas. ¿Cómo podemos hacer precisa la idea de que no hay una teoría físicamente adecuada? ¿Y cuáles serían esos requisitos mínimos de adecuación?. Quizás una respuesta válida para toda teoría no es posible. Sin 
embargo, esta manera de formular la noción de azar objetivo puede hacerse lo suficientemente precisa, por lo menos en algunos casos importantes.

Una de las conclusiones más sobresalientes del análisis filosófico de la física contemporánea es que no hay una teoría física posible que satisfaga un conjunto de condiciones mínimas de adecuación ${ }^{2}$ que permita una descripción determinista de los procesos descritos correcta (pero probabilistamente) por la mecánica cuántica. La mecánica cuántica es pues un contraejemplo para la tesis reduccionista. Por lo menos en el caso de la mecánica cuántica el azar, de acuerdo con la definición anterior, es objetivo.

A través del desarrollo de la interpretación de las probabilidades por frecuencias relativas se ha tratado de formular (implícitamente las más de las veces) una versión diferente del azar objetivo. De acuerdo con esta idea, el azar es objetivo pero su objetividad no proviene del hecho de que el azar es una propiedad física de procesos o sistemas físicos individuales. El azar se concibe en este tipo de propuestas como una propiedad de colectividades.

El núcleo de tales propuestas es la presuposición de que es posible formular una noción de azar objetivo en términos del desorden (medido por medidas matemáticas de desorden) de sucesiones de resultados de observaciones de los procesos físicos. La objetividad del azar se reduciría pues a la objetividad del "desorden" medido por medidas matemáticas apropiadas. De esta manera, la relativización a teorias en la formulación anterior de la noción de azar objetivo sería dispensable. A continuación quiero hacer ver las dificultades que confronta esta versión de la tesis reduccionista del azar. En otros trabajos me dedico a la tarea más positiva de analizar la noción de azar objetivo relativo a teorías (ver Martínez 1990a y 1990b).

3. El azar y la interpretación de las probabilidades por frecuencias relativas. La teoría de frecuencias relativas de Von Mises (tal y como fue desarrollada en Von Mises 1957) es nuestro punto de partida. La teoría de frecuencias relativas de Von Mises parte del hecho de que existen sucesos que se repiten muchas veces, lo que el llama "fenómenos masivos" o "sucesos repetitivos". Sucesos individuales que no se repiten los deja Von Mises fuera del alcance de la teoría de la probabilidad. La base de la teoría es la noción de colectividad. Una colectividad denota una sucesión uniforme de sucesos o procesos que difieren por ciertos atributos observables, color, o número, por ejemplo. Más precisamente, una colectividad para Von Mises (1957) es:

un fenómeno masivo, o simplemente una sucesión numerosa de observaciones que satisfacen las dos condiciones siguientes: (i) La frecuencia relativa del atri-

2 A saber, estas condiciones incluyen leyes de conservación de ciertas cantidades físicas, y la no acción a distancia (localidad). En Cushing y Mcmullin (1989) hay una serie de trabajos recientes que analizan desde diversas perspectivas la significación filosófica de estas condiciones. 
buto observado tenderá a un límite si las observaciones se continuaran indefinidamente. (ii) Este límite no será afectado por ninguna selección de lugar (p. 29).

"Selección de lugar" es el término técnico introducido por Von Mises para denominar el proceso de selección de una subsucesión sobre la única base de su lugar en la serie. Es importante para nosotros hacer énfasis en el papel que juega la restricción a colectividades azarosas. En el caso de colectividades azarosas puede demostrarse que todas las frecuencias relativas permanecen constantes si el conjunto es seleccionado sobre la única base de su lugar en la serie. Esto permite basar la objetividad de las probabilidades sobre la objetividad de la noción de sucesión azarosa.

Después de la introducción del concepto fundamental de colectividad, Von Mises hace ver cómo se pueden especificar las leyes de la probabilidad (axiomas del cálculo de probabilidades) a partir de la postulación de operaciones básicas en colectividades. El cálculo de probabilidades consiste en las transformaciones generadas en estas frecuencias relativas por medio de estas operaciones básicas.

Sin embargo, tal y como Wald y Church mostraron inmediatamente, de acuerdo con la formalización de la idea intuitiva de sucesión azarosa propuesta por Von Mises, no habría prácticamente sucesiones azarosas. ${ }^{3}$ La definición de Von Mises parece que describe una clase vacía, o casi vacía.

Los problemas con la teoría de Von Mises fueron examinados desde distintos puntos de vista. Los filósofos empiristas como Carnap y Reichenbach se concentraron en la dificultad más aparente desde su punto de vista filosófico, la restricción de la teoría a fenómenos repetitivos y a sucesiones azarosas. Ellos trataron de desarrollar una teoría axiomática de la probabilidad que permitiera interpretar las aplicaciones de la teoría sobre la base de una epistemología empirista. Por otro lado, una serie de matemáticos y filósofos trataron de clarificar el aspecto más llamativo de la propuesta de Von Mises, su concepto de sucesión azarosa, que supuestamente permitiría formular una interpretación física clara de la teoría de la probabilidad. Éste es el programa en el que estamos ahora interesados.

Martin-Löf (1966) y otros matemáticos desarrollaron posteriormente modelos matemáticos de sucesiones azarosas que se satisfacen si y sólo si los axiomas de la probabilidad se satisfacen. Esto sin embargo no resuelve el problema de dar una interpretación física del concepto de probabilidad. Para ver esto partamos de un ejemplo simple.

Consideremos el ejemplo paradigmático de un proceso estocástico binario, el lanzamiento de una moneda al aire. Von Mises representa la sucesión de re-

3 Además, las operaciones introducidas por Von Mises no eran condiciones ni suficientes ni necesarias para caracterizar los axiomas del cálculo de las probabilidades. Un estudio de las propuestas de Church y Wald se encuentra en Coffa (1974). 
sultados que el proceso produce de no parar nunca por medio de una función $c \in E \rightarrow(0,1)$, donde $E$ son los enteros positivos. En este caso particular, si definimos $c(i)=1$ si y sólo si el $i$-ésimo lanzamiento (de haber uno) resulta en cara, la probabilidad de cara puede definirse como sigue:

$$
\operatorname{Prob}(\text { cara })=\lim _{n \rightarrow \infty} 1 / n \sum_{i=1}^{n} c(i)
$$

¿Pero cómo están estas probabilidades así definidas relacionadas con las frecuencias relativas obtenidas como resultado de la repetición de un proceso físico particular (del lanzamiento de una moneda por ejemplo)? El establecimiento de una relación clara enfrenta la dificultad siguiente.

Ninguna sucesión de sucesos físicos determina la función $c$ utilizada en la definición de $P$. El meollo de la dificultad es que el concepto de sucesión azarosa propuesto por Von Mises y Wald se aplica sólo a sucesiones infinitas, pero las aplicaciones de la teoría sólo utilizan sucesiones finitas. Si la moneda no se tira no hay forma de saber si el resultado es cara o cruz.

Von Mises en (1957) sugiere que la solución a esta dificultad reside en hacer ver la analogía de una probabilidad con un atributo físico cualquiera, el peso de una mesa, por ejemplo. La idea es la siguiente: la medición de la mesa, nos dice Von Mises, implica en el fondo un proceso de tomar límites. "El valor del peso específico, i.e. el límite del cociente peso/volumen, para un volumen infinitamente pequeño es tan incierto como el valor de la probabilidad derivada de la frecuencia relativa en una sucesión finita de observaciones" (p. 84).

Existe sin embargo una diferencia fundamental en los dos tipos de proceso. En el caso de una serie de mediciones de la mesa hay un sentido intuitivamente claro en el que la sucesión resultante es una sucesión de mediciones del largo de la "misma" mesa. Pero no es lo mismo en el caso de la moneda. Decir que la moneda es la "misma moneda", y que, en tanto que sus características físicas no cambien éste es el referente de la sucesión, es inaceptable para Von Mises. Aquí Von Mises y sus seguidores tendrían que apoyarse sobre una interpretación fisicalista (no meramente extensional) de la probabilidad que Von Mises rechaza por ajena al programa empirista. Para Von Mises, el punto de partida para la clarificación del concepto de probabilidad es el concepto puramente extensional de colectivo, $y$ las series infinitas asociadas con estos colectivos no tienen caracteristicas (matemáticas) que puedan explicar por qué la probabilidad de cara en el quincuagésimo primer lanzamiento es (digamos) 1/2. El apelar a las características físicas de una moneda particular sería apelar a la estructura causal del mundo (como en una intepretación propensista), algo que sería equivalente, a los ojos de un buen empirista como lo era Von Mises, a apelar a la benevolencia divina. Notemos que aquí el problema de fondo es el establecimiento 
de la relación entre la noción matemática de desorden (basada en el concepto extensional de colectividad) y las supuestas aplicaciones físicas.

A menos de que aceptemos un operacionalismo recalcitrante como base de una epistemología científica (en cuyo caso las dificultades de interpretación de la fórmula $P$ simplemente no surgirían), la teoría de Von Mises no parece permitirnos cerrar la brecha entre el concepto de probabilidad física (y el concepto implícito de azar objetivo) y su formulación matemática. La modalidad implícita en la definición de la función $c$ no puede eliminarse de la manera como Von Mises nos sugiere por analogía con la física. La razón de esta deficiencia como hemos visto, y veremos de una manera más general posteriormente, es de fondo.

El programa de Von Mises ha recibido una ayuda importante de parte de una serie de desarrollos de diferentes teorías matemáticas relacionadas con los fundamentos de la probabilidad. Quizás el adelanto más importante parte de la introducción del concepto de "incomprensibilidad" como característica de sucesiones azarosas, introducido por Kolmogorov en los años sesenta (véase Kolmogorov 1969). ${ }^{4}$ A continuación hago un resumen de la teoría de Kolmogorov y de cómo entra ésta en nuestro tema.

4. Sucesiones azarosas como sucesiones incomprensibles. La definición de sucesión azarosa de Kolmogorov descansa sobre la definición previa de complejidad de una sucesión, que tiene importantes aplicaciones tanto en los fundamentos de la probabilidad como en la teoría de la información y en la ciencia de computadores. Parece también ofrecer interesantes posibilidades para la formulación de un cierto tipo de razonamiento inductivo (ver Solomonoff 1964). Para nosotros es sobre todo importante el hecho de que esta definición se aplica a sucesiones finitas y por lo tanto sugiere la posibilidad de superar las dificultades que hemos visto que planteaban las modificaciones de Wald y Church a la definición de Von Mises, que se aplican únicamente a sucesiones infinitas.

Principiemos por formular brevemente la noción básica de complejidad. Comparemos las dos sucesiones siguientes. La primera sucesión consiste de $n$ repeticiones del patrón $01(010101 . .$.$) , la segunda de 2 n$ ocurrencias de 0 y 1 obtenidas por medio de un mecanismo productor de azar (como en el lanzamiento de una moneda). Supongamos que queremos ahora describir instrucciones para reconstruir las dos sucesiones. Intuitivamente es claro que las instrucciones para escribir la primera serán muchísimo más breves que las instrucciones necesarias para escribir la segunda sucesión. En general, las sucesiones azarosas, en el sentido de Kolmogorov, tienen la característica de que el programa mínimo para computarlas (i.e. las instrucciones necesarias para

4 La definición de incomprensibilidad de sucesiones fue propuesta más o menos simultáneamente con fines similares por Chaitin, Solomonoff y Martin-Löf (por lo menos). 
rescribirlas), relativas a un algoritmo dado, es aproximadamente del mismo largo (en bits) que la sucesión misma. La característica de una sucesión azarosa es entonces que no podemos abreviar sustancialmente las instrucciones necesarias para su computación.

En el tratamiento riguroso de Kolmogorov se define primero la idea de "complejidad relativa a un algoritmo" como sigue. Sean $x, y$ etc. variables sobre un dominio de sucesiones finitas o infinitas, sea $\phi$ una variable sobre un dominio de algoritmos, $\phi \in P \rightarrow S$ donde $P$ son las instrucciones para la programación (los programas) y $S$ es el conjunto de sucesiones en cuestión. Definimos

$$
K_{\phi}(x)=\left\{\begin{array}{l}
\min \operatorname{largo}(p), \text { tal que } \phi(p)=x \\
\infty \text { si no existe } p \text { tal que } \phi(p)=x, p \in P
\end{array}\right.
$$

esto es, la complejidad de la sucesión dado el algoritmo es el largo del programa binario mínimo para la sucesión, si este existe. Haciendo uso de la tesis de Church, de acuerdo con la cual las funciones computables por medio de un algoritmo coinciden con las funciones recursivas parciales, podemos identificar $P$ con el conjunto de las funciones recursivas parciales. El lector recordará que las funciones recursivas son aquellas que pueden computarse por medio de una máquina de Turing y que las funciones recursivas parciales son aquellas que no están recursivamente definidas para todo argumento entero.

La definición anterior de complejidad es relativa a un algoritmo. Kolmogorov, sin embargo, ha demostrado un teorema que reduce grandemente esta dependencia.

Teorema (Kolmogorov): Existe una función recursiva parcial $A(p)$ ( $p$ por programa) tal que para cualquier otra función recursiva parcial $\phi$

$$
K_{A}(x) \leq K_{\phi}(x)+C_{\phi}
$$

donde la constante $C_{\phi}$ no depende de la sucesión $x$ a computarse, sino únicamente de la función $\phi$. Las funciones que satisfacen el teorema fundamental son llamadas "asintóticamente óptimas" por Kolmogorov. Éstas son las funciones que caracterizan las sucesiones azarosas. La complejidad de las sucesiones azarosas, medida por cualquier función asintótica optima es apraximadamente dada por el largo de la sucesión.

Indudablemente que este teorema justifica un sentido importante en el que esta definición de Kolmogorov puede considerarse como una descripción de una noción de azar objetivo. Sobre todo en la teoría de la información es claro que (como Kolmogorov y otros han hecho ver) la importancia del teorema en este sentido está fuera de toda duda. Sin embargo, las sugerencias 
de que este teorema fundamenta también la noción de azar objetivo físico (véase por ejemplo Chaitin 1975) deben tomarse con más recelo. Como una propuesta para la caracterización de un concepto de azar físico (objetivo), la definición de sucesiones azarosas de Kolmogorov no parece ir bien encaminada. Schnor (1971) ha hecho ver que la noción estadistica de azar que puede precisarse por medio de pruebas estadísticas efectivas es diferente de la noción de irregularidad o desorden de Kolmogorov. Esto sugiere que la noción de azar como irregularidad (medida por la complejidad) impone requisitos que no tienen significado físico. Una dificultad similar es expresada por Earman (1986, cap. 8).

Otra dificultad es la siguiente. La teoría de Kolmogorov nos proporciona un algoritmo claro para decidir el grado de azar de una sucesión finita. Muestra que con probabilidad muy alta podemos determinar el grado de azar de una sucesión finita. Pero esto no es suficiente para fundamentar la interpretación requerida de la función $c$, por lo menos en tanto que nunca podemos estar seguros de que en nuestro caso particular la sucesión es azarosa y, por lo tanto, nunca podemos estar seguros si tenemos base para predecir, por ejemplo, el quincuagésimo primer lanzamiento a partir de los primeros cincuenta. Hay una probabilidad alta de que esto suceda, pero nada más. Hay también una dificultad que creemos que es todavía más seria que las dos anteriores en relación con el problema de la caracterización del azar objetivo a través de la definición de Kolmogorov a la que no parece habérsele prestado la atención debida. 5

Las funciones que describen procesos físicos (en teorías específicas) son por lo general computables sólo en un sentido generalizado al que la tesis de Church no se aplica. Por lo general la complejidad y el azar surgen en teorías físicas específicas a través de funciones que describen soluciones a ecuaciones diferenciales que son funciones de variables real (o compleja).

El teorema fundamental de Kolmogorov que nos permitúa restringir la relativización de la definición de sucesión azarosa a un lenguaje determinado se aplica a funciones que son computables en el sentido de Turing. Según la tesis de Church, para funciones de variable entera, la clase de las funciones computables según Turing es la clase de las funciones efectivamente computables por medio de un algoritmo. En el caso de funciones de variable entera existe un único concepto, universalmente aceptado, de computabilidad. Pero en el caso de funciones de variable real la situación es muy diferente. Hay diferentes propuestas para la formulación de una extensión del concepto de computabilidad elemental (Turing) a funciones de variable real. Es bastante conocida la propuesta de Grzegorczyk (1955) pero no existe, ni parece plausible, la

5 Véase sin embargo Hellman 1981. 
existencia del tipo de coincidencia de diferentes criterios de computabilidad expresada en la tesis de Church. ${ }^{6}$

Es claro pues que, en los casos en los que la tesis de Church no es aplicable, no existe una generalización del teorema de Kolmogorov que nos permita acotar la relativización a un lenguaje determinado del concepto de desorden de una sucesión. Una función azarosa en nuestro lenguaje (teoría) puede resultar ordenada en otro lenguaje (teoría) y no tiene que haber ni siquiera una constante que acote la diferencia (como el teorema de Kolmogorov nos lo asegura para funciones recursivas parciales).

Estas dificultades nos llevan a la conclusión de que la noción de azar matemático caracterizado por medio de una medida de complejidad de las sucesiones debe desecharse como una caracterización de la idea de azar físico. Más en general, las dificultades que hemos encontrado con la caracterización de la noción de azar requerido por la interpretación frecuentista de Von Mises nos hacen ver una dificultad de fondo en el intento empirista de caracterizar el azar físico en términos de una noción matemática de azar. Las dificultades con la propuesta de Von Mises y sus sucesores, así como con la definición de Kolmogorov, sugieren que el concepto de azar físico no puede reducirse a una medida del orden de sucesiones generadas sin tomar en cuenta para nada aspectos físicamente relevantes del mecanismo de generación de las sucesiones. De alguna manera, el hecho de que la moneda es la "misma" moneda debe incorporarse en la explicación física de lo azaroso de una sucesión de lanzamientos. Pero esto requiere entonces que abandonemos el presupuesto de que el azar físico puede reducirse a una medida de desorden matemático y tratemos de caracterizar el azar como un aspecto de la estructura causal del mundo.

\section{REFERENCIAS BIBLIOGRAFICAS}

Aberth, O., 1980, Computable Analysis, Mcgraw Hill, N. York.

Chaitin, G. J., 1975, "Randomness and Mathematical Proof", Scientific American, vol. 232, 5 (mayo, 1975).

Coffa, A., 1974, "Randomness and Knowledge", en K. Schaffner y R. Cohen (eds.), PSA 1972, Boston Studies in the Philosophy of Science, vol. 64 (Reidel, 1974), pp. 103115.

Cushing, J. y E. McMullin (eds.), 1989, Pbilosopbical Consequences of Quantum Theory, Notre Dame Press.

Earman, J., 1986, A Primer on Determinism, Reidel.

6 Una exposición de los esfuerzos por generalizar la noción de computabilidad en el sentido de Turing a los reales puede encontrarse en Aberth (1980). 
Hellman, G., 1981, "Randomness and Reality", PSA 1978, vol. II, P. Asquith e I. Hacking (Comps.).

Kolmogorov, A. N., 1969, "On the Logical Foundations of Information Theory and Probability Theory", en Problemy Peredachi Informatsii, vol. 5, No. 3.

Martin-Löf, P., 1966, "The Definition of a Random Sequence", Information and Control 9, pp. 602-619.

_ 1970, "On the Notion of Randomness", en A. Kino (ed.), Intuitionism and Proof Theory, Nort Holland.

Martínez (1990a), "La objetividad del azar en un mundo determinista", Crítica, agosro 1990.

- (1990b), “¿Que es una ley estadística irreducible?”, sometido a publicación. Dictomen pendiente.

Von Mises, R, 1957, Probability Statistics and Truth, Dover, N. York. Traducción al inglés del original alemán publicado en 1932.

Nagel, T., 1939, "Principles of the Theory of Probability", vol. 1, núm. 6 de International Encyclopedia of Unified Science, Stanford University Press.

Popper, K., 1962, "The Propensity Interpretation in the Calculus of Probability, and the Quantum Theory", en S. Körner (ed.), Observation and Interpretation in the Philosophy of Physics, Nueva York, Dover.

- 1982, The Open Universe, Rowman and Lirtlefield.

Rogers, H., 1967, Theory of Recursive Functions and Effective Computability, McGraw Hill, Nueva York.

Schnorr, C., 1971, Zufälligkeit und Wahrscheinlichkeit, Springer Verlag, Nueva York.

Solomonoff, R. J., 1964, “A Formal Theory of Inductive Inference”, Parte I, Information and Control, 7, 1-22.

Stegmüller, W., 1973, Probleme und Resultate der Wissenschafttheorie und analytischen Pbilosoplice, tomo IV, Springer Verlag, Berlín. 